Hydrol. Earth Syst. Sci., 11, 1243-1247, 2007

www.hydrol-earth-syst-sci.net/11/1243/2007/

(C) Author(s) 2007. This work is licensed

under a Creative Commons License.

\title{
Technical Note: Water flow routing on irregular meshes
}

\author{
D. Bänninger \\ Institute of Environmental Geosciences, University of Basel, Bernoullistrasse 30, 4056 Basel, Switzerland
}

Received: 14 August 2006 - Published in Hydrol. Earth Syst. Sci. Discuss.: 11 December 2006

Revised: 6 February 2007 - Accepted: 13 April 2007 - Published: 3 May 2007

\begin{abstract}
For spatially explicit hydrological modelling an algorithm was required that works as a cellular automata on irregular meshes. From literature it was found that the usual algorithms applied for this purpose do not route the water flow correctly between adjacent cells. In this study the hydraulic linking between mesh cells is done by calculating the flow cross section between the mesh cells. The flow cross sections are positioned in the centre of the mesh edges and are perpendicular to the local gradient of the digital elevation model. The presented algorithm is simple in its implementation and efficient in computation. It is shown that the proposed algorithm works correctly for different synthesised hill slope shapes.
\end{abstract}

\section{Introduction}

In this study we present a spatially explicit hydrological model running on irregular meshes. Models running on irregular meshes are rarely used for spatially explicit hydrological models, probably because flow routing is not trivial on irregular meshes, as stated in Grayson and Blöschl (2000). Moreover, existing models based on regular or irregular meshes are often physically imprecise with respect to water flow routing between mesh cells. Nevertheless, the use of irregular meshes is of high interest because they are qualified for describing real terrain shapes very efficiently. However, so far the effect of triangulated terrain resolution on basin hydrologic response has received surprisingly little attention (Vivoni et al., 2005).

Triangular irregular meshes are applied for basin delineation to route the runoff through a watershed using kinematic cascades (Palacios-Velez and Cuevas-Renaud, 1986; Palacios-Velez et al., 1998). Irregular meshes are used to

Correspondence to: D. Bänninger

(dominik.baenninger@unibas.ch) generate runoff networks by analysing whether the slopes of two neighbouring cells with a common edge forms a ridge, channel or plane (e.g., Gandoy-Bernasconi and PalaciosVelez, 1990). Another model using irregular meshes was presented by Ivanov et al. (2004) and Vivoni et al. (2005). The flow paths are calculated - as in many other approaches along the steepest direction between triangle centres or triangle edges (Tucker et al., 2001; Ivanov et al., 2004); the nodes of the triangulated meshes are used to describe the conditions in the respective Voronoi cell.

Approaches for flow routing on irregular meshes are derived from hydrological models running on regular meshes. Common approaches are: (i) single direction flow based on the highest altitude difference between cells (O'Callaghan and Mark, 1984), (ii) random flow direction with a probability distribution proportional to altitude difference between the centre and neighbouring cells (Fairfield and Leymarie, 1991), and slope direction (Lea, 1992; Costa-Cabral and Burges, 1994; Tarboton, 1997); (iii) multiple direction flows weighted according to the slopes to the adjacent mesh cells (Quinn et al., 1991; Freeman, 1991; Wigmosta et al., 1994). On irregular meshes various algorithms such as draining a cell to its lowest neighbour (e.g., O'Callaghan and Mark, 1984) and weighting the outflow from a cell to its neighbours according to the altitude difference (e.g., Fairfield and Leymarie, 1991) are not suitable because the orientation of the triangle edge with respect to the flow direction must be taken into account.

In this study the water flow routing is derived by first calculating the flow gradient vector within each mesh cell. Since triangulated meshes are used, the shape of a mesh cell is given by three points which define a plane. The slope of the plane is the gradient of the triangle. The new idea proposed in this study is that, as a second step, the projection of the triangle edges on the perpendicular of the gradient vector is calculated. The length of the projected triangle edges corresponds to the flow cross section between two adjacent

Published by Copernicus GmbH on behalf of the European Geosciences Union. 


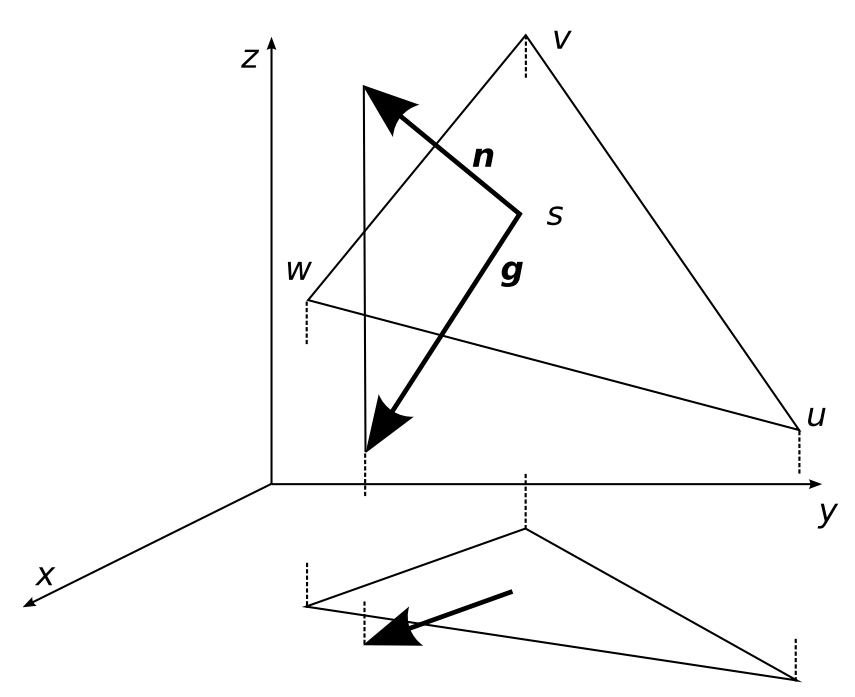

Fig. 1. Derivation of the slope gradient for a single triangle. The horizontal projection of the triangle is added to the sketch for illustration.

triangles. Thus, this approach can be understood as a discretisation of the space by flow cross sections which are positioned in the centre of each mesh edge. The orientation of the cross sections is defined by the triangle slopes.

For the presented approach it is assumed that there is only lateral water movement, i.e. water inflow (e.g., rain) and water outflow (e.g., percolation into subsoil layers) are not considered. Thus, in general terms, this approach is suitable for any lateral distribution problem on an irregular mesh where the gradients of the mesh topology points into direction of the driving forces.

\section{Theory}

\subsection{Mesh generation}

The program Triangle (Shewchuk, 1996) is used in this work to generate triangulated irregular meshes. Triangle performs a Delaunay triangulation which means that the nodes of the triangulated mesh are arranged in such a way that every circle passing through three points of a triangle will encompass no other points (Delaunay, 1934). A property of the Delaunay triangulation is the minimisation of the maximum internal angle. Nevertheless, in Triangle a flag can be set to restrict the minimum angle to a given value. To be sure that the generation of flat and long triangles is supressed, this flag is set to $20^{\circ}$.

Discretisation of the space with triangulated irregular meshes is useful because the resolution of such meshes can be variable in space, linear elements in the landscape can be represented well, and the number of nodes is drastically reduced compared to regular meshes (Lee, 1991; Braun and Sambridge, 1997). Palacios-Velez and Cuevas-Renaud

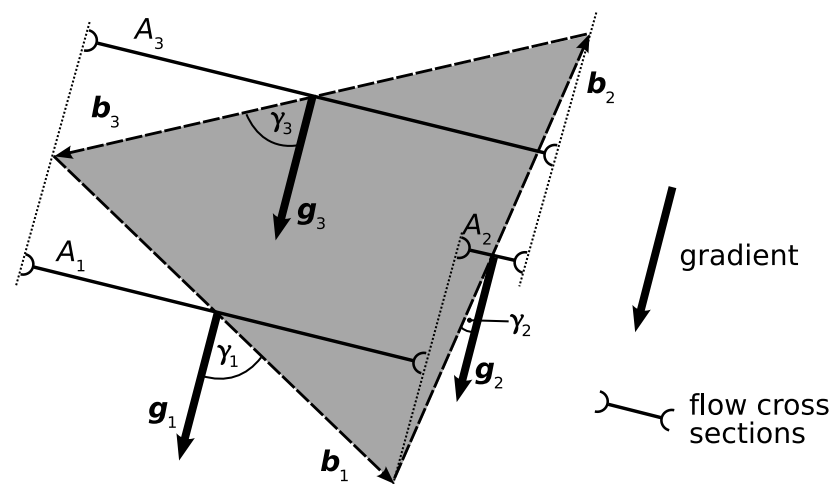

Fig. 2. Scheme for derivation of flow cross sections. This figure shows a triangle which is surrounded by other triangles lying in the same plane.

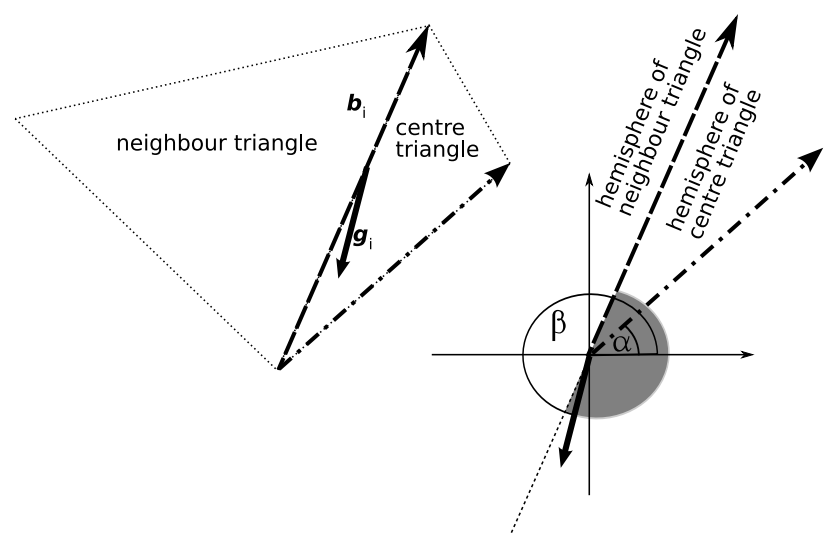

Fig. 3. Identification of the triangle to which the gradient points. The gray semicircle labels the side of the centre triangle.

(1986) reported that a regular mesh needs 14 to 250 times more nodes for one node needed in an irregular mesh.

\subsection{Mesh topology}

The description of the mesh topology is extended by calculating the local gradients for each triangle and the projection of the triangle edges on the perpendicular to the local gradient.

The gradient is calculated by using the following procedure: Each triangle is defined by three nodes, denoted here as $u, v$, and $w$. The normal vector $\boldsymbol{n}$ towards the $(u, v, w)$ plane is given by the vector product of two vectors describing two edges of a given triangle. The next step is to move the normal vector so that it starts in the centroid $s$ of the triangle. Next, the projection in $z$-direction of the normal vector onto the $(u, v, w)$-plane is calculated. The projection is then the gradient $\boldsymbol{g}$ of the $(u, v, w)$-plane (cf. Fig. 1).

To calculate the flow across the edges of two adjacent triangles, the mean value of the gradients for each of the two triangles is taken. The flow cross section $A_{i}$ for the $i$-th triangle edge ( $i=1,2$ or 3$)$ is defined by the projection of the 


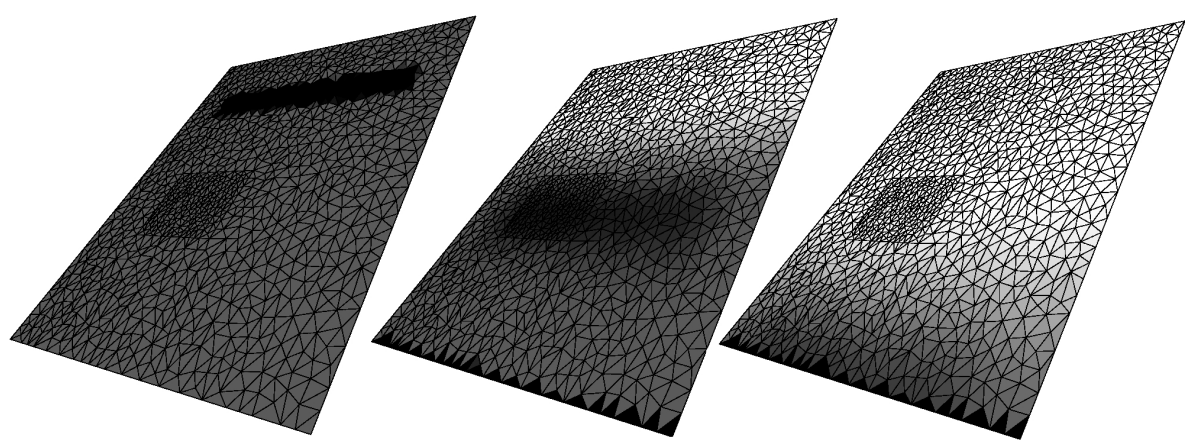

Fig. 4. Water flow on a triangulated irregular mesh describing an inclined plane. The three images show the soil moisture (white $=$ dry, black $=$ wet) at the beginning and after 20 and 40 time steps, respectively.

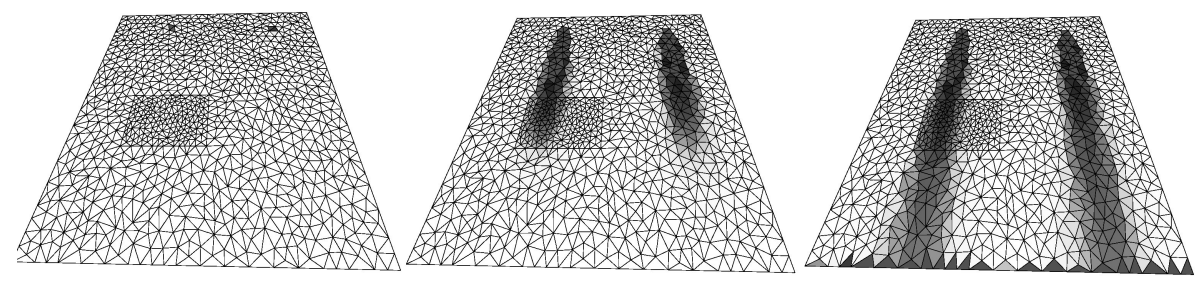

Fig. 5. Water flow from two sources on triangulated irregular mesh describing an inclined plane. The three images show the soil moisture (white $=$ dry, black $=$ wet) at the beginning and after 30 and 60 time steps, respectively.

common triangle edge onto the perpendicular of the gradient: $A_{i}=\left|\boldsymbol{b}_{i}\right| \cdot \sin \gamma_{i}$ where $\boldsymbol{b}_{i}$ is the vector of the common triangle edge and $\gamma_{i}$ the angle between gradient $\boldsymbol{g}_{i}$ and $\boldsymbol{b}_{i}$. The angle $\gamma_{i}$ is found by the scalar product of $\boldsymbol{g}_{i}$ and $\boldsymbol{b}_{i}$ (cf. Fig. 2).

In order to determine whether the gradient vector points towards the centre triangle, i.e. the triangle currently under observation, or towards the neighbouring triangle, the following procedure is used (cf. Fig. 3):

- Find an angle range that describes the hemisphere of the centre triangle.

- Calculate the angle $\alpha$ of the vector pointing into the direction of the third polygon node of the centre triangle.

- Calculate the angle $\beta$ of the gradient.

- If the angles $\alpha$ and $\beta$ are in the range of the centre triangle hemisphere, there is inflow from neighbour to centre triangle. Outflow from centre to neighbouring triangle is given in all other cases.

The three flow cross sections belonging to a given triangle have an important property: In case the centre triangle and the three adjacent triangle lie in the same plane, the length of inflow cross sections is equal to the length of the outflow cross sections. Thus, in case the hydraulic properties at each edge of the center triangle are equal, the in- and outflow of water balances to zero for the center triangle.

\subsection{Flow calculation}

The purpose of calculating water flow in this study is for the validation of water flow routing with time. The simple relation

$O=k \cdot A \cdot z_{d} \cdot t \cdot \theta$

is taken to describe outflow from a triangle, where $O$ is the outflow, $k$ is the hydraulic conductivity, $z_{d}$ is the depth of the soil, $t$ is the duration of the time step, and $\theta$ is the soil saturation degree. The time step $t$ has to be so small that no triangle gets completely drained in one single time step.

Equation (1) describes the driving forces for water movement very rough: Water flow in dependence of gravitation is lumped within the parameter $k$, and water flow induced by soil water potential is approximated by water content differences. Nevertheless, this equation can be used for our case because the slopes in each digital elevation model is constant. The implementation of more sophisticated flow equations is straight forward.

\section{Results}

The first simulation calculates the water drainage on an inclined plane. The initial conditions were a wet restricted area on top of the plane surrounded by soil of a lower constant soil moisture. The mesh discretisation contains a rectangular sub-area that has a finer resolution than its neighbourhood. 

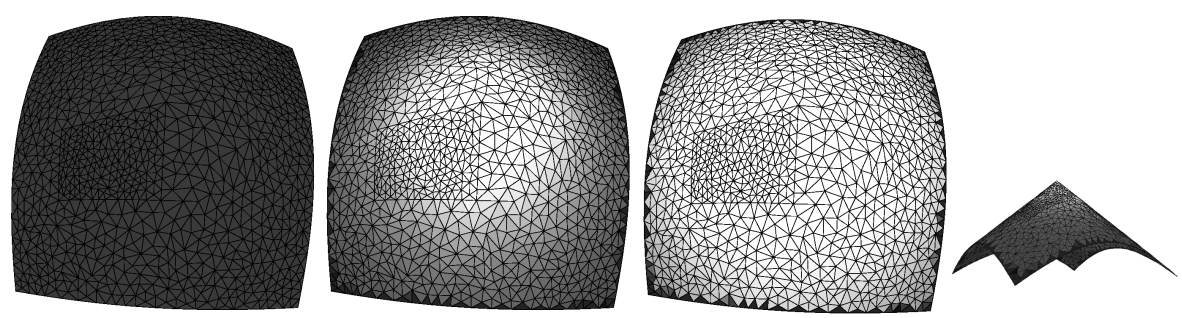

Fig. 6. Water flow on the same triangulated irregular mesh as use for the previous simulations, but now stretched over a cone. The first three images show the soil moisture (white $=$ dry, black $=$ wet) at the beginning and after 10 and 20 time steps, respectively. The cone is observed from the top. The most right image is a side view of the cone.

Figure 4 shows the soil moisture distribution at the beginning, in the middle and the end of the simulation run. In this as well as in the following simulations presented here no mass transfer through the outside boundary of the mesh was allowed.

In the second simulation run the routing of water from one single triangle is analysed. Two water sources are defined, i.e. the two triangles are continuously supplied with the same amount of water. Figure 5 depicts instantaneous snapshots of simulations of the soil moisture distribution on the mesh below the two water sources.

In the last simulation presented here, it is analysed whether the flow on curved hill slopes spreads regularly. The mesh stretched over a cone - is initialised with constant soil moisture. Figure 6 depicts the drainage starting at the top of the cone moving regularly downwards.

\section{Conclusions}

From the results presented we can qualitatively conclude that the water flow routing over the irregular mesh runs correctly and the velocity of water flow routing is independent of the mesh resolution. Qualitative comparison of these results with the results of frequently used algorithms (D8, Multi flow direction method, Lea's method, DEMON, D $\infty$; summarized in Tarboton (1997)) illustrates that the presented algorithm is more accurate than those presented in Tarboton (1997): It is demonstrated by simulation with the cone digital elevation model that flow routing is independent on the flow direction, a typical weakness, e.g., in the D8 algorithm of O'Callaghan and Mark (1984).

From the simulation run that imposes two sources on top of the inclined plane, it can be seen that the water flow paths disperse. The spreading of the flow stream depends on the resolution of the triangulated mesh: It is smaller within the high resolution area than in the coarse resolution area (Fig. 5). This result is similar to the findings of Tarboton (1997).

\section{Summary}

An approach is presented which defines the routing of water flow on triangular meshes generated with Delaunay triangulation. The water flow in space is described by flow cross sections which are distributed in space; they have an orientation perpendicular to the local gradient. The connectivity of the flow cross sections is defined by the edges of the triangulated mesh. Simulations indicate that the presented method is a suitable approach to flow routing on irregular meshes. Thus, this approach is a step towards addressing multi spatial scale hydrological problems.

Edited by: D. Solomatine

\section{References}

Braun, J. and Sambridge, M.: Modelling landscape evolution on geological time scales: A new method based on irregular spatial discretization, Basin Research, 9, 27-52, 1997.

Costa-Cabral, M. and Burges, S.: Digital elevation model networks (DEMON): A model of flow over hillslopes for computation of contributing and dispersal areas, Water Resour. Res., 30, 16811692, 1994.

Delaunay, B.: Sur la sphere vide, Bulletion of Academy of Sciences of the USSR, pp. 793-800, 1934.

Fairfield, J. and Leymarie, P.: Drainage networks from grid digital elevation models, Water Resour. Res., 27, 709-717, 1991.

Freeman, T.: Calculating catchment area with divergent flow based on a regular grid, Comput. Geosci., 17, 413-422, 1991.

Gandoy-Bernasconi, W. and Palacios-Velez, O.: Automatic cascade numbering of unit elements in distributed hydrological models, J. Hydrol., 112, 375-393, 1990.

Grayson, R. and Blöschl, G.: Spatial Patterns in Catchment Hydrology, Cambridge University Press, 2000.

Ivanov, V., Vivoni, E., Bras, R., and Entekhabi, D.: Catchment hydrologic response with a fully distributed triangulated irregular network model, Water Resour. Res., 40, w11102, doi:10.1029/2004WR003218, 2004.

Lea, N.: Overland Flow: Hydraulics and Erosion Mechanics, in: An aspect driven kinematic routing algorithm, edited by: A. Parsons and A. Abrahams, 1992. 
Lee, J.: Comparison of existing methods for building triangular irregular network models of terrain from grid digital elevation models, International Journal of Geographical Information Systems, 5, 267-285, 1991.

O'Callaghan, J. and Mark, D.: The extraction of drainage networks from digital elevation data, Computer Vision Graphics Image Processes, 28, 328-344, 1984.

Palacios-Velez, O. and Cuevas-Renaud, B.: Automated rivercourse, ridge and basin delineation from digital elevation data, J. Hydrol., 86, 299-314, 1986.

Palacios-Velez, O., Gandoy-Bernasconi, W., and Cuevas-Renaud, B.: Geometric analysis of surface runoff and the computation order of unit elements in distributed hydrological models, J. Hydrol., 211, 266-274, 1998.

Quinn, P., Beven, K., Chevallier, P., and Plachon, O.: The prediction of hillslope flow paths for distributed hydrological modeling using digital terrain models, Hydrol. Processes, 5, 59-80, 1991.
Shewchuk, J. R.: Triangle: Engineering a 2D Quality Mesh Generator and Delaunay Triangulator, in: Applied Computational Geometry: Towards Geometric Engineering, edited by: Lin, M. C. and Manocha, D., Vol. 1148 of Lecture Notes in Computer Science, Springer-Verlag, from the First ACM Workshop on Applied Computational Geometry, pp. 203-222, 1996.

Tarboton, D.: A new method for the determination of flow directions and upslope areas in grid digital elevation models, Water Resour. Res., 33, 309-319, 1997.

Tucker, G., Lancaster, S., Gasparini, N., Bras, R., and Rybarczyk, S.: An object-oriented framework for distributed hydrologic and geomorphologic modeling using triangulated irregular networks, Computer and Geosciencies, 27, 959-973, 2001.

Vivoni, E., Ivanov, V., Bras, R., and Entekhabi, D.: On the effects of triangulated terrain resolution on distributed hydrologic model response, Hydrol. Processes, 19, 2101-2122, 2005.

Wigmosta, M., Vail, L., and Lettenmaier, D.: A distributed hydrology-vegetation model for complex terrain, Water Resour. Res., 30, 1665-1679, 1994. 